\title{
Coordinated Multicast/Unicast Transmission on 5G: A Novel Approach for Linear Broadcasting
}

\author{
David Jiménez-Soria $^{1} \cdot$ Francisco J. Martín-Vega ${ }^{1} \cdot$ Mari Carmen Aguayo-Torres ${ }^{1}$ (D)
}

Accepted: 16 August 2021 / Published online: 16 September 2021

(c) The Author(s) 2021

\begin{abstract}
Linear broadcasting services, with a scheduled programming, constitute a paramount telecommunication service for today's society. Although the existing technology is mature, current linear broadcast systems have serious limitations when providing service to moving users or users placed in areas with complex orography and poor signal quality. To overcome these limitations, 3GPP 5G standard has included a work item to support 5G multicast/broadcast services for future Release 17. This paper investigates the integration of point-to-point (unicast) communication with cellular multicast/broadcast on $5 \mathrm{G}$ technology to extend the current support of linear broadcasting services. This integration relies on the use mobile edge computing (MEC) at the $5 \mathrm{G}$ base station (gNB) to host a dynamic adaptive streaming over HTTP (DASH) server that is coordinated with the multicast transmission to complement the broadcast service. This approach join the reliability of point-topoint communications, with dedicated resources for each user, with the spectrum efficiency of multi-cast communications, where a set of users share common resources. The cooperation between those unicast and multicast schemes allows those users whose coverage is not good enough, to complete the linear broadcast flow through the point-to-point transmission via MEC. The benefits of such approach have been assessed with simulations in a realistic scenario that considers a vehicle moving across a sparsely populated region in southern Spain. Results reveals that throughput and bitrate playback (reproduction rate) are greatly improved when unicast/multicast integration is enabled since the number of stalling events is reduced significantly.
\end{abstract}

Keywords Linear broadcasting $\cdot 5 \mathrm{G} \cdot \mathrm{EMBMS} \cdot \mathrm{MEC} \cdot \mathrm{DASH}$

\section{Introduction}

The Third Generation Partnership Project (3GPP) 5G new radio (NR) standard, that was initially defined with Release 15 , aims at supporting a plethora of diverse scenarios and use cases while reducing operational cost and easing network operation and maintenance [1]. This has been achieved with the design of a flexible radio access network (RAN) that adds

Mari Carmen Aguayo-Torres

aguayo@ic.uma.es

1 Dpto. de Ingeniería de Comunicaciones, Universidad de Málaga, Málaga, Spain 
innovative concepts as scalable numerology, bandwidth parts, hybrid precoding or dynamic Time Division Duplexing (TDD) among others that cope with frequency bands ranging from $410 \mathrm{MHz}$ to $52.6 \mathrm{GHz}$. With the recent completion of Release 16 on June 2020, the set of scenarios considered has been extended beyond cellular networks to include vehicular networks, unlicensed spectrum and industrial internet of things [2]. Nevertheless, the focus of these two releases have been unicast transmissions services on $5 \mathrm{G}$.

Release 17, which is expected to be completed by June 2022, has included a work item aiming to support broadcast/multicast transmissions on $5 \mathrm{G}$. This will bring the wide flexibility and efficiency of $5 \mathrm{G}$ networks to linear broadcasting services to greatly improve user experience while reducing operational costs. In addition, $5 \mathrm{G}$ broadcast/multicast services can complement conventional broadcasting technology which has severe deficiencies in some scenarios like mobility or users in remote areas. Conventional broadcasting technology is based on single frequency network (SFN) where a limited set of high power high towers (HPHTs) offers nation wide linear broadcasting services, e.g., digital terrestrial television (DTT). Although the technology is mature it has serious limitations with users that are moving or are placed in regions with complex orography and coverage problems. Besides of this, there are sparsely populated areas where upgrading the broadcasting network is often not profitable. Nevertheless, in these areas, a cellular network has also been deployed in recent years. Its coverage, maturity, and robustness can be used to offer linear broadcasting with high definition content.

The multimedia broadcast/multicast service (MBMS) was first added in the 3GPP standard in 2005 (Release 6) to optimize the distribution of simulcast (i.e., broadcast and multicast) services via the 3rd generation cellular systems. The MBMS standard, covering terminal, radio, core network, and user service aspects, evolved in Release 9 into enhanced MBMS (eMBMS), built on top of the Long Term Evolution (LTE) technology.

In LTE, the simulcast transmission of data over the radio access network can be carried out using either single-cell point to multipoint (SC-PTM) or MBMS over single frequency networks (MBSFN) deployment.

MBSFN exploits the orthogonal frequency division multiplexing (OFDM) radio interface to send simulcast data as a multicell transmission over a synchronized SFN. Although it increases the signal to interference and noise ratio (SINR) due to the absence of inter-cell interference, it avoids sending different content to users located at other cells.

SC-PTM uses the MBSFN system architecture with small changes on the existing radio and core network infrastructures and protocols but follows a different approach in the allocation of radio resources aimed at improving the radio efficiency as well as at reducing the latency. The granularity of the simulcast services is down to a single cell so that its coverage area can be dynamically adjusted cell by cell according to the distribution of users. Moreover, those resources unused by the simulcast transmission can be allocated to single users similarly to the usual unicast procedures (instead of a fixed allocation during deployment) [3].

The latest improvement on simulcast services came on 2017 with Release 14 for LTE, which was referred as further evolved multimedia broadcast multicast service (FeMBMS) or enhanced TV (EnTV). This was the first release that incorporated a transmission mode to deliver content from existing HPHTs broadcast infrastructure, it also increased the intersite distance for SFN and it allowed allocation of all available carrier bandwidth to broadcast service.

Although, the existing support of LTE to simultcast services is noticeable, the increased flexibility, reduced latency, and wider bandwidth that brings 5G NR compared to LTE have motivated a number of recent works that investigates different options to support broadcast/ 
multicast services with 5G technology. In [3], a design of NR MBMS for terrestrial broadcast is proposed with minor modifications on current $5 \mathrm{G}$ standard. It is demonstrated with simulations that proposed design for NR MBMS outperforms latest enhancements of MBMS for LTE related to Release 14. Several architectural and functional improvements, to support dynamic selection between unicast and multicast/broadcast transmission modes on 5G NR are presented in [4]. In [5] potential solutions to enable dynamic and seamless switching between unicast and multicast, both in the downlink and the uplink are discussed and assessed via simulations. The switching between unicast and multicast is based on the number of users that aim at receiving multicast services, and involve a reconfiguration of radio bearers to change from unicast bearers to multicast bearers and vice versa. An adaptive error recovery using unicast transmission on a proxy server that complements the multicast transmission on LTE is proposed on [6]. Simulation were carried out for urban scenario using results from OPNET [7] and a model developed on MATLAB.

The present paper explores modifications to current $5 \mathrm{G}$ standard to support multicast/ broadcast services, and thus, it presents a possible variant of the current standard. These modifications consider current LTE cellular multicast functionality SC-PTM as a framework. Nevertheless, contrary to other works for 5G like [3-5], the focus is to overcome known limitations of existing terrestrial broadcasting technology, i.e., the support of moving users and the support of users placed at areas with complex orography. This work considers unicast transmission to handle errors in multicast communication as presented in [6] for LTE; however our proposal considers a mobile edge computing (MEC) system that hosts a dynamic adaptive streaming over HTTP (DASH) server instead of a proxy server. In addition, we propose the use terrestrial broadcast infrastructure to reduce the backhaul traffic. MEC enables cloud computing capabilities at the edge of the cellular network, reducing network congestion and improving application performance as related processing tasks run closer to the cellular client. The DASH server distributes via unicast transmission those segments lost by a user, complementing the eMBMS transfer. Jointly employed, unicast and multicast transmissions reduce the stalling probability for those users whose coverage is not good enough to complete the linear broadcast flow. To asses the proposal, simulations use a highly accurate channel model that captures the effects of irregular terrain using real topographical data. Obtained results demonstrate the benefits of the proposal to reduce the stalling events.

The rest of this paper is organized as follows. Firstly, Sect. 2 presents an overview of the 3GPP support for broadcast/multicast services with LTE and the main modifications proposed for $5 \mathrm{G}$. Then, Sect. 3 describes the joint unicast/multicast transmission and the enabling technology elements. Section 4 describes the simulated scenario and presents numerical results. Finally, Sect. 5 concludes the key findings.

\section{Multicast Transmission on 3GPP Cellular Networks}

Broadcast is a transport technology to deliver the same content to an unlimited number of devices with a defined quality of service, without increasing substantially network capacity requirements, energy consumption, or costs.

Cellular systems are commonly used for unicast transmissions. In this mode, a dedicated channel is established with each user equipment (UE). In scenarios with a large number of users or devices consuming the same data, the use of simulcast transmission can offer substantial capacity gains, ensuring a cost-effective and high-quality delivery mechanism [8]. 
At unicast transmission, required radio resources grow linearly with the number of UEs receiving the same data. Resource allocation efficiency is improved by simultaneous transmission of data to a set of users (simulcast). Note that via simulcast transmission, all users receive the same signal. Thus the reachable transmission rate is mainly enforced by the UE with the worst radio conditions.

Simulcast services over cellular networks can be classified as broadcast and multicast. Via broadcast services, all users receive the same information within the service area. In multicast services, users have to subscribe to the specific service before they can receive the information. While broadcast communication is unidirectional, multicast users can establish a return channel allowing interactivity with the network. This return channel can also be used to subscribe to the desired service.

\subsection{MBMS Evolution on LTE}

There are two main approaches for MBMS services on LTE: MBSFN and SC-PTM.

\subsubsection{Multi-cell PTM: MBSFN}

MBSFN is a simulcast transmission technique introduced to support the transmission of eMBMS over LTE networks. MBSFN exploits the LTE's OFDM radio interface to send simulcast data as a multi-cell transmission over a synchronized SFN.

A common time-synchronized waveform is transmitted simultaneously from a set of evolved nodes B (eNBs) over the same frequency resources. Data is scheduled using an ad-hoc physical channel with extended cyclic prefix (CP) known as Physical Multicast Channel (PMCH) [3]. The transmissions from the multiple cells are sufficiently tightly synchronized for each to arrive at the UE within the OFDM CP so as to avoid intersymbol interference (ISI). In effect, this makes the MBSFN transmission appear to a UE as a transmission from a single large cell, dramatically increasing the SINR due to the absence of inter-cell interference. Radio cells that shall transmit the same content to multiple users forms a so called MBSFN area. Multiple cells can belong to such an area, and every cell can be part of up to eight MBSFN areas. There could be up to 256 different MBSFN areas defined, each one with an own identity. Once deployed, MBSFN areas do not change dynamically.

The principal enhancements to enable this service are summarized below:

- Release 9 introduced eMBMS by adding new radio bearers, logical and physical channels and new logical entities, like the broadcast multicast service center, which is the entry point for content and is responsible for user authentication. With this release transmissions occupy the entire system bandwidth, and multiplexing between multicast and unicast in the same subframe is not allowed [8].

- Release 10 allowed the use of unused MBSFN subframes for unicast reception and introduced a RAN-based counting of UEs in connected mode which are interested in an MBMS service.

- Release 12 introduced MBMS operation on demand which allowed seamless service activation and deactivation based on UE's service compsumtion reporting. This release also added improvements in the physical measurements (e.g., signal power, error rates). In addition, the UEs can be ordered to perform for MBSFN network optimization. 
- Release 14 introduced MBSFN for V2X communications. This release also added the support of large geographical areas, covering an entire country, with a maximum cell radius up to $100 \mathrm{~km}$. A receive only mode called free-to-air was introduced, which allows reception with no need of an uplink (UL) or SIM card, thus receiving without user registration in the network [3].

\subsubsection{Single-Cell PTM (SC-PTM)}

Release 13 introduced SC-PTM as part of eMBMS aiming to reduce the latency and improve the efficiency in radio resource allocation. SC-PTM make use of the eMBMS system architecture used for MBSFN with small changes on the existing radio and core network infrastructure and protocols; but it follows a different approach in the allocation of radio resources. The granularity of the broadcast/multicast services is down to a single cell so that the broadcast/multicast area can be dynamically adjusted cell by cell according to the distribution of users. SC-PTM allows one cell to broadcast the same content to a group of UEs multiplexing broadcast and unicast data on the same Physical Downlink Shared Channel (PDSCH). The multicast transmissions are scheduled using a common Radio Network Temporary Identity (RNTI) for a group of users. This approach is more agile than the one followed by MBSFN which involves a different and dedicated physical channel, the $\mathrm{PMCH}$, with static resources being assigned.

This transmission mode was further extended with Release 14, which introduced SCPTM for V2X, SC-PTM for internet of things (IoT) and NarrowBand IoT (NB-IoT).

\subsection{MBMS on 5G NR}

5G adds paramount innovations compared to previous $4 \mathrm{G}$ networks that are specially appealing for MBMS services. Main innovations are summarized below:

- Scalable numerology: 5G is based on OFDM as LTE; however contrary to the previous standard, 5G allows the selection of the Sub-Carrier Spacing (SCS), i.e., numerology, to adapt to the link and radio conditions but also to reduce the latency [9]. A higher SCS leads to a more robust transmission to phase noise and other impairments; but also it reduces the symbol and slot duration, which reduces the latency. Nevertheless, a shorter symbol duration might provoke ISI if the delay spread of the channel is higher than the cyclic prefix.

- Bandwidth adaptation: The available carrier bandwidth is logically split in bandwidth parts (BWPs). It can be defined up to 4 BWPs, but only one can be active at a time. The switch of the active BWP is indicated via Physical Downlink Control Channel (PDCCH). BWPs allows a efficient use of available bandwidth and save power [10].

- Dynamic TDD: Besides the downlink (DL) and UL slots, 5G introduces the flexible slots which can be either DL or UL and are not determined a priory. Besides, the selection of slots as DL or UL is more dynamic with $5 \mathrm{G}$ than with previous standards and allows the definition of mixed slots with some symbols allocated to UL, some being flexible, and some allocated to DL [9].

- Hybrid precoding: This approach enables efficient implementation of massive multiple input multiple output (MIMO) in 5G, splitting the precoding between analog and digitial domains. It is considered RF beams, that are conformed with analog precoding which is handled separately than digital precoding [11]. 
- Improvements on higher layers: Besides physical layer, some improvements are added to higher layers as the introduction of a new layer named Service Data Adaptation Protocol (SDAP), which is used for packet marking with Quality of Service (QoS) flow identifiers and mapping of these flows to radio bearers. In addition, the concatenation of packets takes place with $5 \mathrm{G}$ on Medium Access Control (MAC) layer instead of in Radio Link Control (RLC) layer as in LTE, which leads to a smaller latency in 5G [5].

There a number of modifications proposed to include MBMS on 5G, with the two existing approaches: SC-PTM and MBSFN. The former would require the introduction of a new RNTI type, to allow the group based resource allocations. In this approach, a group of users would be associated to the same group RNTI value, and hence a single transmission on PDCCH would signal a multicast transmission on PDCCH. This saves overhead signalling on PDCCH and transmission resources on PDSCH. On the other hand, inclusion of MBSFN would require the intoduction of new negative numerologies, to use smaller SCS that support longer inter-site distances SFN operation [5].

\section{Coordinated Multicast/Unicast Transmission}

In this section, the integration of multicast and unicast transmission to provide a reliable and high definition linear broadcast service thought a 5G network is described. Two emerging tools that enable the proposed method are presented first, then the details of the proposal are described.

\subsection{Emerging Tools}

Two main elements expected to be deployed to integrate broadcast/multicast transmission schemes in $5 \mathrm{G}$ are described below.

The MEC technology is designed to be implemented in cellular base stations (or other edge nodes) to allow a flexible and fast implementation of new applications and services. MEC technology is not exclusive to $5 \mathrm{G}$ but is undoubtedly integral to its efficiency. MEC permits low latency, high bandwidth and real time access to the 5G RAN information by authorized third parties, such as application developers and content providers. 5G standard define the enablers for edge computing, allowing MEC and 5G to collaboratively route traffic.

For sure, streaming is one essential service for 5G. In contrast to fixed rate streaming, adaptive streaming detects content consumers' capabilities and adapts the video resolution based on user throughput. The objective is to provide the best possible quality according to the particular context of each client.

The current most important example of adaptive streaming is DASH, an International Organization for Standardization (ISO) standard for streaming live and on-demand content. DASH uses HTTP for data transmission and is based on segmentation of multimedia files.

In DASH, a multimedia file is encoded in different resolutions that are divided into small portions called segments. The client selects the resolution of the next segment to be downloaded. The duration of video segments is fixed while their size in bits varies depending on the resolution that the client selects. Downloaded sequentially and played in order, the result is playback without interruptions (if the download does not suffer setbacks). Adaptation logic is managed by the client implemented at the customer device. Changes in 
segment resolution levels are decided after calculating their expected download times after throughput prediction.

\subsection{Proposed Multicast and Unicast Integration}

The block diagram of the proposed system, along with the relationship between different components is illustrated in Fig. 1. Multicast information is carried out over a point to multipoint transmission. Functionality equivalent to eMBMS takes form on a $5 \mathrm{G}$ NR radio interface. Moreover, unicast communication is carried out directly between each user and the $5 \mathrm{G}$ base station $(\mathrm{gNB})$ as needed.

The video representation function is responsible for generating the video segments that are requested later by the eMBMS and the DASH servers. These segments are generated from broadcast multimedia content, however there are two options to receive such content. One is to receive it over existing 5G backhaul connections, which are normally implemented with fiber optics links (blue arrow in the figure); although wireless self-backhauling is also supported by $5 \mathrm{G}$. The other approach, is to use a terrestrial broadcast receptor of the linear broadcast service already provided by HPHTs to offload the backhaul connection (purple arrow in the figure). This later approach has the benefit of reusing the existing linear broadcast network that is being complemented or enhanced with the $5 \mathrm{G}$ network. The reasoning is that the receptor of terrestrial broadcast service connected to the gNB can be more sophisticated than the one placed on the user's devices. In addition, since this receptor will be placed at a fixed location, the terrestrial broadcast reception will be better than the reception of moving users. In any case, if the reception of such a signal is not possible, the delivery of multimedia content to the gNB must be done using the backhaul connections.

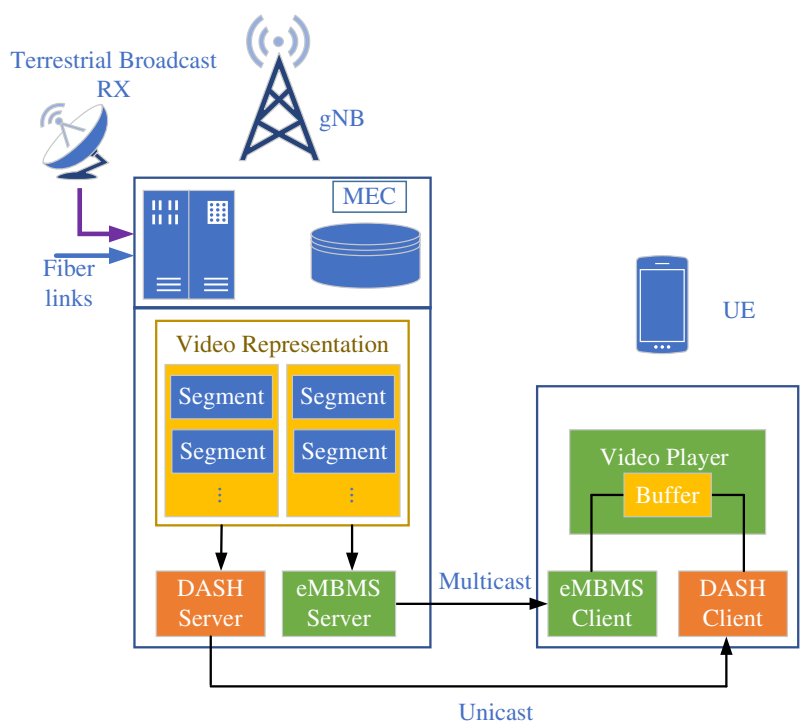

Fig. 1 Block diagram of the proposed coordinated multicast and unicast system 
The generation of video segments with different resolutions must be time aligned to combine them in a single video playback. In this work, two resolutions are considered: high and low. The eMBMS server transmits high video resolution segments to all multicast users. Transmission is carried out with the SC-PTM framework as described in Sect. 2. Then, the eMBMS client receives the transmitted video segments. In case that a given segment is lost, it is indicated to the video player.

The video player for a user keeps the received segments in a buffer. If only multicast transmission were employed, when some segments were lost because the channel decoder cannot correct the errors, multicast throughput would fall, and stalling occur. Nevertheless, with the proposed coordinated unicast transmission integrated with the multicast transmission, the video player sends to the DASH client an indication to request a lower resolution video for lost segments.

DASH client at the user device supports the broadcast service if the throughput is not enough to reproduce the video. In that case, the DASH client requests a video stream with a lower resolution to allow the video player to continue showing the content.

As shown in Fig. 1, the DASH server is hosted at the MEC. It is responsible for distributing via unicast the video adapted to the available throughput to those users requiring it. The DASH server receives the HTTP request for a lost segment from a player and selects a multimedia representation whose bitrate fits the available unicast throughput for the terminal sending the request. Regarding the unicast bit rate allocated to each client, the DASH server obtains it from the base station.

\section{Performance Evaluation}

\subsection{System Modelling}

Figure 2 shows the LTE SC-PTM resource grid in a subframe $(1 \mathrm{~ms})$, which is the same as that for unicast transmission as both share resources. LTE employs OFDM at the physical layer. An OFDM symbol is formed by the combination of a set of $N$ subcarriers equally-spaced in frequency $\Delta f$ to form an array of parallel signals. A cell-specific reference signal (C-RS) is also transmitted (marked in orange in the figure) to allow channel

Fig. 2 Resource block frame structure for LTE SC-PTM. In orange, cell specific reference signal elements; in white: resource element allocatable for unicast or multicast data symbols

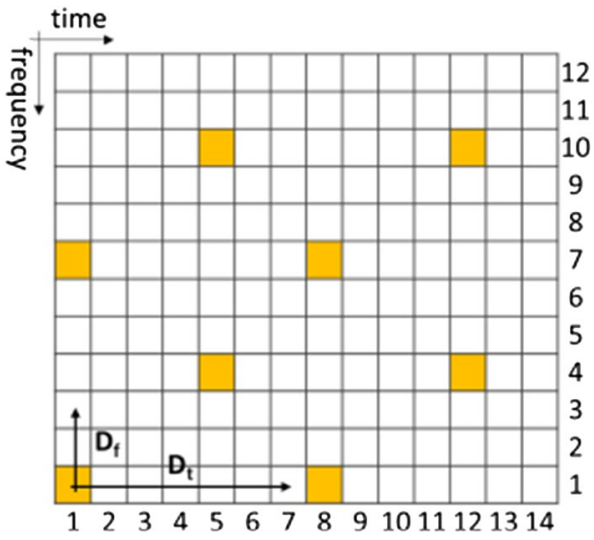


estimation. Other resource elements (REs) can be allocated to data (left in white). Minimum allocatable resources is a resource block (RB), formed by 12 subcarriers along a subframe.

$5 \mathrm{G}$ transmission technologies are very similar to those of LTE. Besides channel coder, the main difference is that 5G NR supports variable OFDM numerology (i.e, SCS) and carrier frequency range (i.e., frequency range 2 for frequencies above $6 \mathrm{GHz}$ and frequency range 1 otherwise). Each numerology is labeled as a parameter $\mu$. The numerology $\mu=0$ represents $\Delta f=15 \mathrm{kHz}$, which is the same as LTE. This value has been considered here.

The primary transmission unit in 5G NR is the slot. The resource grid in one slot is very similar to that in LTE. In our approach, unicast and multicast data can be multiplexed over the same resources, in a similar way to LTE SC-PTM transmission.

For unicast transmission, each data subcarrier is modulated using a coded QAM modulation selected depending on the SINR reported by the specific user the data belongs to. Basically, the adaptive modulation and coding (AMC) algorithm decides the higher modulation and coding scheme (MCS) in order to keep the block error rate (BLER) below a target which depends on the service. For video transmissions it is fixed to $B L E R_{T}=0.1$ [12]. Note that data segments might be lost with a certain probability depending on the current radio link state. Resources are allocated to users by the scheduler, located at the base station. A round-robin scheduling algorithm has been employed in this work. The actual throughput depends on both, the number of allocated RBs, which can change from one slot to the next one, and on the spectral efficiency of the employed MCS.

For multicast transmission, the same data is sent to a group of UEs. The same MCS has to be employed for all users. In order that all users reach the target BLER, that lowest SINR reported by the set of multicast users determines the employed MCS.

Figure 3 represents the Cumulative Density Function (CDF) of the effective SINR that is used to select the MCS for multicast transmission to a group of users as a function of the size of the group (i.e., the number of users). As it can be observed, the more users form the broadcast group, the more likely it is to obtain a smaller SINR, and thus a smaller throughput due to AMC. This has an effect similar to reducing the coverage area of the cell for the broadcast service. It is also observed that, through a unicast connection, we always obtain higher values of SINR since this value now only depends on a single user.

Fig. 3 Equivalent SINR for diferents broadcast groups of user

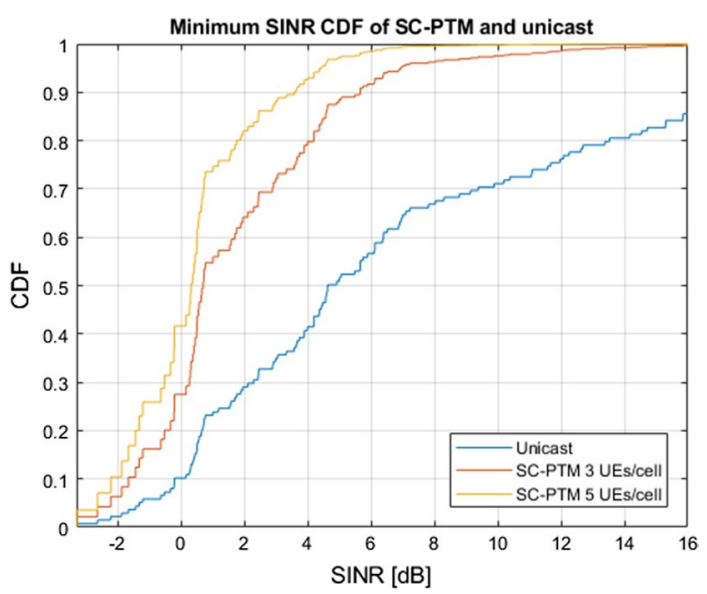




\subsection{Simulated Scenario}

We have simulated over a sparsely populated area with a complex orography for DVB-T coverage. The chosen area has been a section of road A-4 between Linares and Manzanares, as shown in Fig. 4. Twelve real gNBs with two cells per site has been placed as given in [13] at that area. Five users uniformly placed move along the road at a fixed speed in a random direction.

Users are served by that site whose signal is received with maximum average power. Longley-Rice propagation model has been used to determine the path loss between the site and the user. The Irregular Terrain Model (ITM) also includes free-space loss, terrain diffraction, ground reflection, refraction through the atmosphere, tropospheric scatter, atmospheric absorption, and buildings. With those parameters as given in Table 1, and real topographical data from that route, the average SINR at each point in the area is shown in Fig. 4. Higher values of SINR are represented with warm colors whereas lower values are represented with cold colors. It can be noted that the SINR is higher on valleys with line of sight with the serving base station, whereas valleys without line of sigh due to the orography of the terrain have very small SINR. In general, the SINR tends to be higher at higher locations since base stations are also placed at high locations and then it is more likely to have a line of sight. As mentioned above, it should be noted that for the multicast transmission, the equivalent SINR is that minimum for all users.

The test environment is based on the guidelines defined in Report ITU-R M.[IMT-2020. EVAL] for Rural-eMBB (configuration A) in table 8-2 c [14]. The test environment includes a custom antenna array that is also simulated. Regarding fading, a TDL-A wideband channel with delay and Doppler spread as given in Table 1 has been considered. Resources per slot allocated to simulcast transmission allows transmitting $125 \mathrm{kB}$.

Regarding available video resolutions, two bitrates are supported in our simulations. The high resolution video sent by multicast should be played back at $4.7 \mathrm{Mbps}$. A low

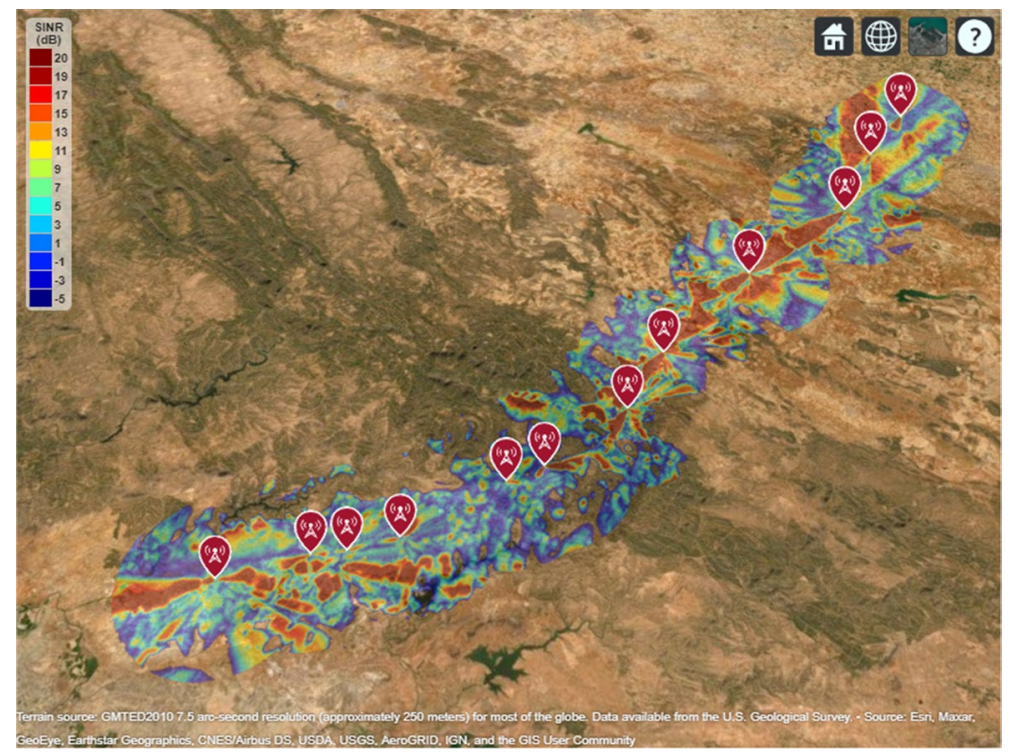

Fig. 4 SINR over the road map 
Table 1 Values for transmission parameters

\begin{tabular}{lll}
\hline Parameter & & Default value \\
\hline gNB & Antenna height & $35 \mathrm{~m}$ \\
& Total transmit power & $49 \mathrm{dBm}$ \\
& Number of antenna elements & $8-\mathrm{by}-8 \mathrm{URA}$ \\
& Antenna element gain & $8 \mathrm{dBi}$ \\
OFDM & Carrier frequency & $700 \mathrm{MHz}$ \\
& Bandwidth & $30 \mathrm{MHz}$ \\
& Subcarrier Spacing & $15 \mathrm{KHz}$ \\
Channel & Frequency Doppler & $70 \mathrm{~Hz}$ \\
& Delay spread & $200 \mu \mathrm{s}$ \\
UE & Speed & $80 \mathrm{~km} / \mathrm{h}$ \\
& Noise figure & 7 \\
& Antenna element gain & $0 \mathrm{~dB}$ \\
& Antenna height & $1.5 \mathrm{~m}$ \\
& Thermal noise level & $-174 \mathrm{dBm} / \mathrm{Hz}$
\end{tabular}

resolution version ( $0.808 \mathrm{Mbps}$ playback bitrate) of the same video is available by unicast transmission. The segment characteristics, taken from [15] for $1 \mathrm{~s}$ segment duration, are shown in Table 2. Video player queue is set to 50 Mbytes.

\subsection{Simulation Results}

Figure 5 shows the bitrate of one representative UE player together with the throughput that the user receives along the route. While the segments received via multicast are correctly decoded, the high resolution video version is played back at 4.7 Mbps. When needed, flow switches to unicast transmission and low $0.8 \mathrm{Mbps}$ bitrate through the assistance of the DASH server located at the MEC. Stalling occurs only when bitrate decreases to 0 as the unicast transmission cannot carry out the $808 \mathrm{kbps}$ transmission.

As a result of the cooperation between multicast and unicast transmission, most unicast communications are carried out for user positions at which the SINR is below $12 \mathrm{~dB}$ while multicast communication is enough above $25 \mathrm{~dB}$. With this diversification, it is sought that both transmissions can maintain the ideal data flow to avoid stops in reproduction.

To show the improvement due to unicast, we compare the bitrate with and without DASH support in Fig. 6. We observe how the number of stallings is significantly reduced by changing the video bitrate to $808 \mathrm{kbps}$ with the support of the DASH server, resulting in improved quality of experience during video playback.

In Fig. 5 we observed how the throughput has drops to 1 Mbps where the DASH will be working adapting the video flow to the available bandwidth.

Table 2 Video resolutions [15]

\begin{tabular}{llll}
\hline Resolution & Pixels & Video bitrate & Segment size $(\mathrm{kB})$ \\
\hline Low & $1280 \times 720$ & $808 \mathrm{kbps}$ & 101.18 \\
High & $1920 \times 780$ & $4.7 \mathrm{Mbps}$ & 565 \\
\hline
\end{tabular}


Fig. 5 Bitrate playback and throughput with DASH support

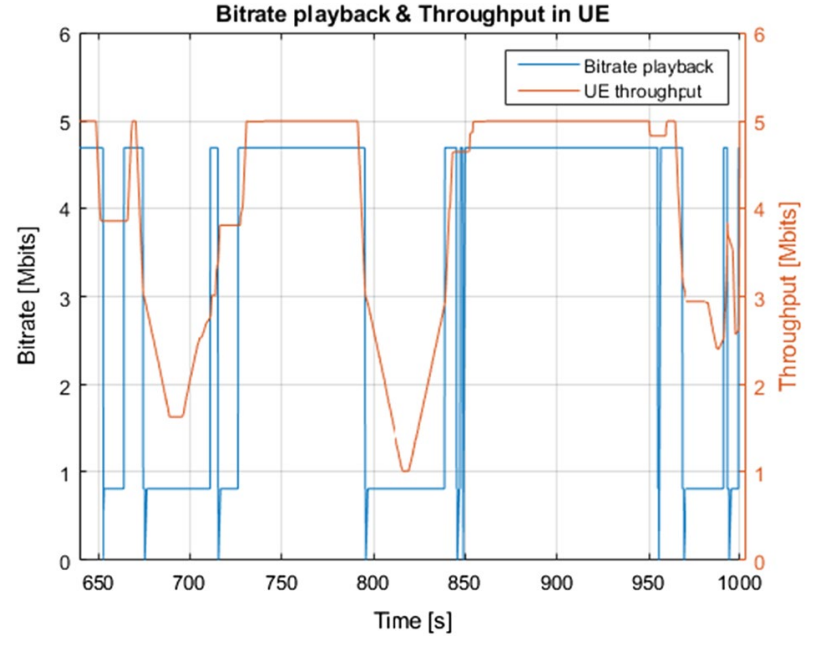

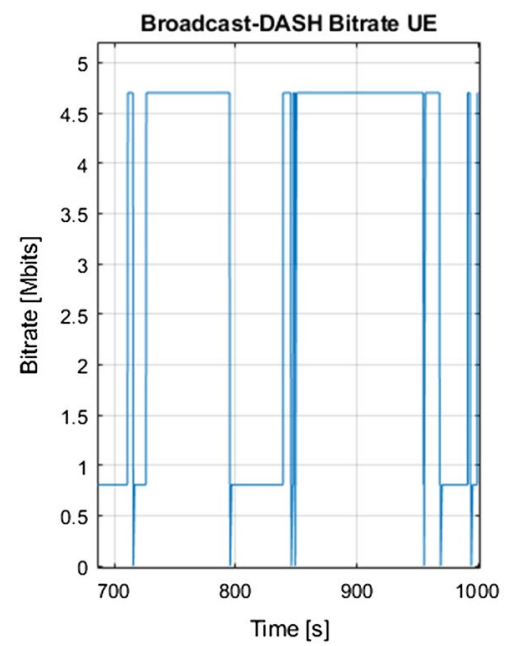

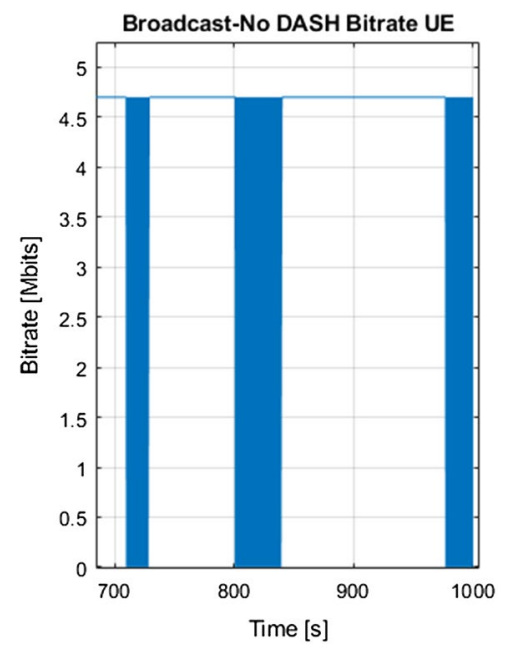

Fig. 6 Comparison between integrated unicast/multicast (left) and multicast only (right)

\section{Conclusions}

This paper first gives a brief overview of existing support for multicast services with LTE networks, and main changes expected for its possible introduction on $5 \mathrm{G}$ with next release 17.

Then, using SC-PTM as a framework, is has been explored the use of a promising approach that join unicast and multicast transmission on 5G networks to bring linear audiovisual content to areas where the terrestrial broadcast network has severe limitations to provide stable and reliable service.

In this proposal, a multicast service, based on the SC-PTM, transmits the same signal to a group of users associated to a given cell, avoiding the redundant use of resources to send 
the same information. In addition, a DASH server implemented via MEC allows adjusting the video stream resolution to the available throughput at the user. A reliable service with unicast transmission of the missing segments improves the quality of user experience during video playback.

Finally, the proposed coordinated unicast/multicast transmission is assessed with realistic simulations that captures the irregularity of the terrain using real topographical data from a route in the southern of Spain. The simulated scenario considers moving users through the motorway that connects two cities in the considered region. It has been shown that with this diversification, stalls in reproduction can be significantly reduced. The proposed SC-PTM technology can be employed simultaneously to regular cellular communication, thus giving a new use to the $5 \mathrm{G}$ infrastructure in areas without good broadcasting coverage.

Acknowledgements This work has been partially supported by Radio Televisión Española through Impulsa Visión RTVE grant and by the Universidad de Málaga. We are grateful to Pere Vila, Esteban Mayoral Campos, Adolfo Muñoz Berrón and Miguel Ángel Bona San Vicente for their support and collaboration during the development of the project.

Funding Open Access funding provided thanks to the CRUE-CSIC agreement with Springer Nature. Funding for open access charge: Universidad de Málaga / CBUA.

Open Access This article is licensed under a Creative Commons Attribution 4.0 International License, which permits use, sharing, adaptation, distribution and reproduction in any medium or format, as long as you give appropriate credit to the original author(s) and the source, provide a link to the Creative Commons licence, and indicate if changes were made. The images or other third party material in this article are included in the article's Creative Commons licence, unless indicated otherwise in a credit line to the material. If material is not included in the article's Creative Commons licence and your intended use is not permitted by statutory regulation or exceeds the permitted use, you will need to obtain permission directly from the copyright holder. To view a copy of this licence, visit http://creativecommons.org/licenses/by/4.0/.

\section{References}

1. Farooq, M., Waseem, M., \& Qadri, M. T. (2017). Understanding 5G wireless cellular network: Challenges, emerging research directions and enabling technologies. Wireless Personal Communications, 95, 261-285.

2. Parkvall, S., Blankenship, Y., Blasco, R., Dahlman, E., Fodor, G., Grant, S., et al. (2020). 5G NR release 16: Start of the 5G evolution. IEEE Communications Standards Magazine, 4(4), 56-63.

3. Gimenez, J. J., Carcel, J. L., Fuentes, M., Garro, E., Elliott, S., Vargas, D., et al. (2019). 5G new radio for terrestrial broadcast: A forward-looking approach for NR-MBMS. IEEE Transactions on Broadcasting, 65(2), 356-368.

4. Säily, M., Estevan, C. B., Gimenez, J. J., Tesema, F., Guo, W., Gomez-Barquero, D., \& Mi, D. (2020). $5 \mathrm{G}$ radio access network architecture for terrestrial broadcast services. IEEE Transactions on Broadcasting, 66(2), 404-415.

5. Garro, E., Fuentes, M., Carcel, J. L., Chen, H., Mi, D., Tesema, F., et al. (2020). 5G mixed mode: NR multicast-broadcast services. IEEE Transactions on Broadcasting, 66(2), 390-403.

6. Lentisco, C. M., Bellido, L., \& Pastor, E. (2017). Seamless mobile multimedia broadcasting using adaptive error recovery. Mobile Information Systems, 2017(2), 1-12.

7. "OPNET. Riverbed Technology". Available at tiny.cc/OPNET. Last access on 28 March 2021.

8. Gomez-Barquero, D., Navratil, D., Appleby, S., \& Stagg, M. (2018). Point-to-multipoint communication enablers for the fifth generation of wireless systems. IEEE Communications Standards Magazine, 2(1), 53-59.

9. 3GPP, Technical specification group radio access network; NR; physical channels and modulation (release 16), TS 38.211, Rev. 16.4.0, Jan 2021. 
10. 3GPP, Technical specification group radio access network; NR; physical layer procedures for data (release 16). In 3rd Generation partnership project (3GPP) TS 38.214, Rev. 16.4.0, Jan 2021.

11. Onggosanusi, E., Rahman, M. S., Guo, L., Kwak, Y., Noh, H., Kim, Y., et al. (2018). Modular and high-resolution channel state information and beam management for $5 \mathrm{G}$ new radio. IEEE Communications Magazine, 56(3), 48-55.

12. Ruiz-Sicilia, J. C., Aguayo-Torres, M. C. (2020). Logistic regression for BLER prediction in 5G. In XXXV Simposio Nacional de la Unión Científica Internacional de Radio, URSI 2020.

13. "CellMapper". www.cellmapper.net. Last access on 29 December 2020.

14. ITU-R. Draft new report ITU-R M.[IMT-2020.EVAL]: Guidelines for evaluation of radio interface technologies for IMT-2020 (2017).

15. Vaca-Rubio, C. J., Gómez, G., Aguayo-Torres, M. C., López Martínez, F. J. (2019). Statistical characterization of the chunk size distribution in DASH. In Actas del XXXIV Simposio Nacional de la Unión Científica Internacional de Radio (URSI 2019) (2019).

Publisher's Note Springer Nature remains neutral with regard to jurisdictional claims in published maps and institutional affiliations.

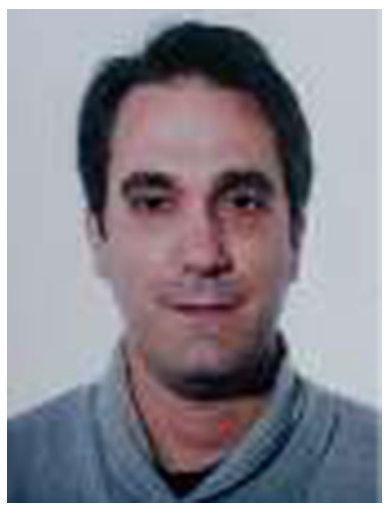

David Jiménez-Soria received the M.S. degree in Telematics and Telecommunication Networks from the Universidad de Málaga (Spain) in 2020. He is currently a researcher at the Department of Communications Engineering. His research interests include applications of artificial intelligence to wireless networking, machine learning for communications, and rapid prototyping by software radio.

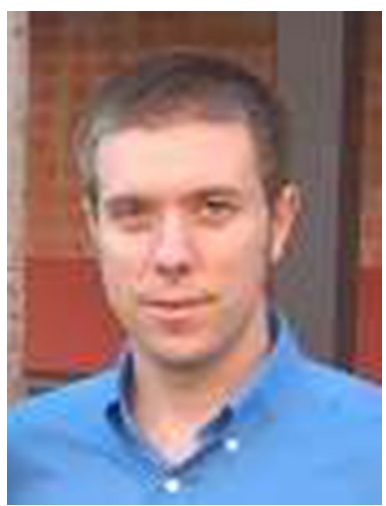

Francisco J. Martin-Vega received the Ph.D. degree in Telecommunication Engineering from the University of Malaga, Spain, in 2018 (M.S. 2012). From 2011 to 2017 he was an associate researcher at the University of Málaga, where he participated in contracts with several industry partners related to cellular and satellite communications. He joined Keysight Technologies as a wireless engineer. Recently he joined the Department of Communications Engineering at University of Malaga as a postdoc researcher. His research activity has focused on the mathematical modeling of communication systems. 


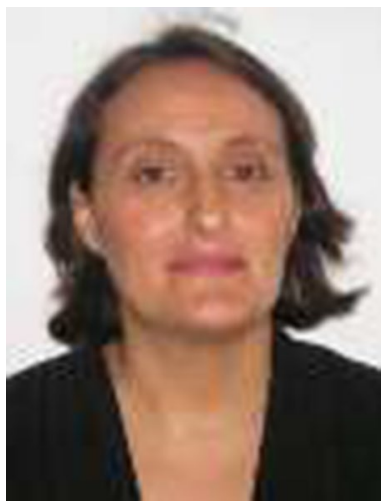

Mari Carmen Aguayo-Torres received the Ph.D. degree in Telecommunication Engineering from the University of Malaga, Spain, in 2001 (M.S. 1994) with a thesis on Adaptive OFDM. She is currently Head of the Department of Communications Engineering at University of Malaga. Her main research interests are vertical-aware 5G \& 6G technologies with probabilistic QoS guarantees. Her current work includes theoretical analysis with tools such as stochastic geometry besides statistical evaluation of real and simulated networks. Dr. Aguayo-Torres is involved in a number of public and private funded projects and actively collaborates with industry, mainly in the field of wireless communications. 